\title{
SHORT CRACK PROBLEMS IN GAS TURBINE DISKS
}

J. Drexler and J. Statečny

Aeronautical Research and Test Institute, Prague, Czechoslovakia

ABSTRACT

Gem turbine disks operat ing under low-cycle fatigue conditions represent typical nechine parts with short crack systems. Herewith, the authors aim at presenting nom methods being good for solving real problems with short cracks in the process of disk airworthiness certification.

KEYWORDS

Wechenical systems; machine parts; gas turbine disks; low-cycle fatigue; crack dotecting and development; short crack problems.

BACKGROLND

Wino; Drexler (1984) stated that there is no universal method which would enable solving short crack problems in machine parts under all possible varleties of structural configurations and corresponding operational conditions. Obviousiy, the eng imeering solutions of the mentioned problems are to be searched for a specific inchine type and a representative sample of its working fleld characteristics, only. iypleal subproblems of

1. detecting a short crack at its first occurence,

1. detectling a short crack at its first occurence, 2. an adequate doscription of a short crack system

crack character

3. est imating the safe life or life to safe crack occurence in the morst danag fir-tree blade attachment on the disk taken for being the least rel lable disk in the chole envisaged product ion series.

In this relation, it is the authors task to present some possible solutions of the ubproblems mentioned above.

PROBLEM OF DETECT ING A SHORT CRACK AT ITS FIRST OCCURENCE

The main problem in examining a disk under test - should any of the known detection whod be used - is the human operator taking the following two final resolutions: 
i) the crack is ( is not ) present in the investigated zone,

ii) if present, the crack length est imation is $L$ mm.

The presence of the human operator in crack detecting procedure forces the necessity of carefully verifying outside reference data on crack detecting probability before applying them to one $s$ own purpose. One of the reliable (robust) methods of ten used independently on others under laboratory conditions is visual detection supported by several component penetrants and a microscopo in this sual detection supporis necessitated hom to quantify the crack detection abil lity level of a hu method such quant if icat ion is required by civil Aviation Authorities inel a human operator

First, let us examine the information structure of the results of human operator's activity in detecting cracks: these results are in form of registered crack length data referring to critical zones, e.g. edges of disk blade at tachments, see Fig. 1 . Such a crack length datum forcibly involves the following information

a) the one of the type of critical machine part under investigation,

b) the one of the type of the detection method used, i.e. on human ability ( one operator or operator group ) to crack detection,

c) the one of a finished programme test unit number,

d) the one of crack trace length as accessible to visual investigation,

a) the one on all other operating conditions which were not explicitely mentioned.

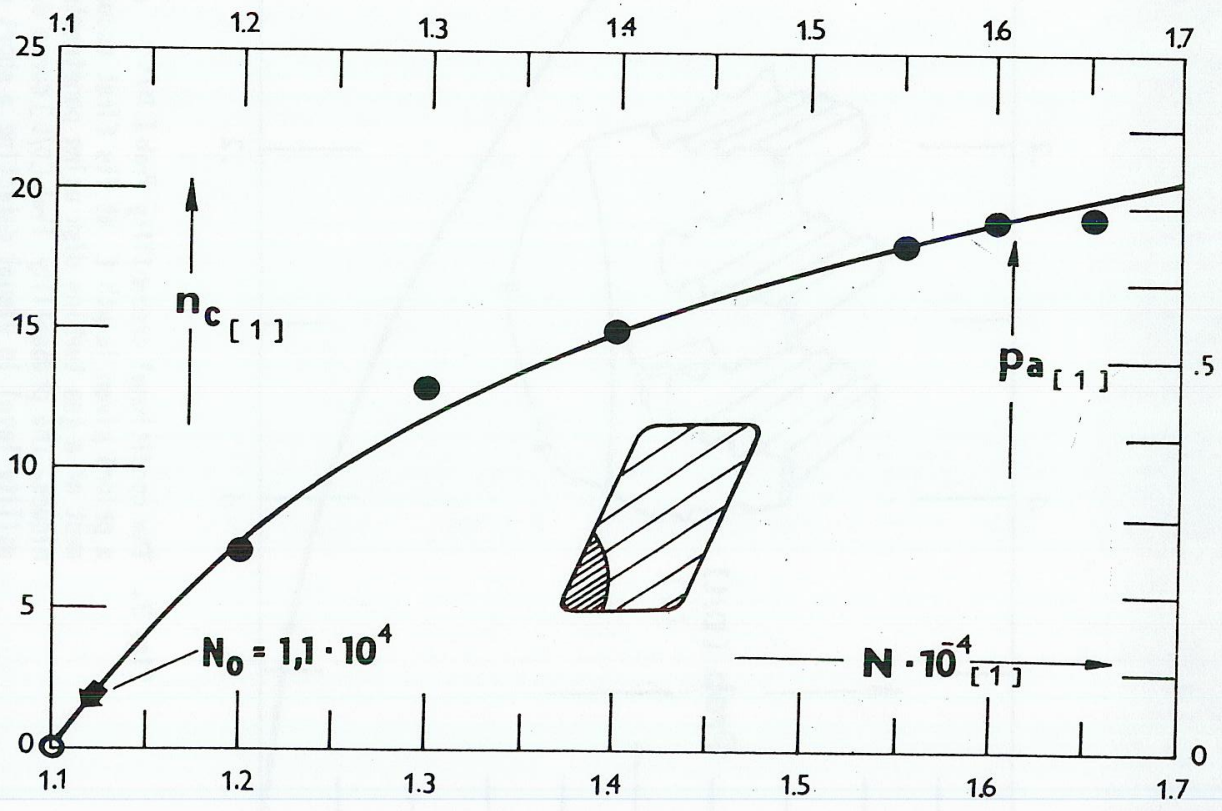

Fig. 1. Crack number $n_{c}$ in function of the programme test unit number $N$ as found on disk blade at tachment edges, back side ( Němec, Drexler, 1984 ). Pa - crack occurence probability in a fir-tree blade attachment.
The whole information content of a crack length datum may be depleted by the folloing elementary statement def initions:

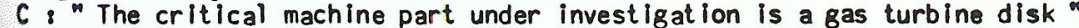
N: " The programme test unit number finished by the disk up to this inspection time Instant is $N$

O: "Cracks are visually detected by a selected human operator whose abllity level Is to be quantified "n

i: "The crack trace length is $L$ mm"

$O$ : "Other aperating condition present when detecting cracks"

In our case, we have limited considerations of a specific machine part (disk), of an a priori agreed programme test unit number and of standard laboratory conditions. Hence, the fact that a crack length datum involves information expressed through statements $C$ to $O$ can be registered by a composed statement ( $D_{0} L / C C_{0} N_{0} O$ ) being of random character due to the problem nature.

Therefore, our task to quantify the operator's ability level in respect to crack detection is transferred to that one of establishing an adequate probabilistic characleristic. Using the product rule, we may write

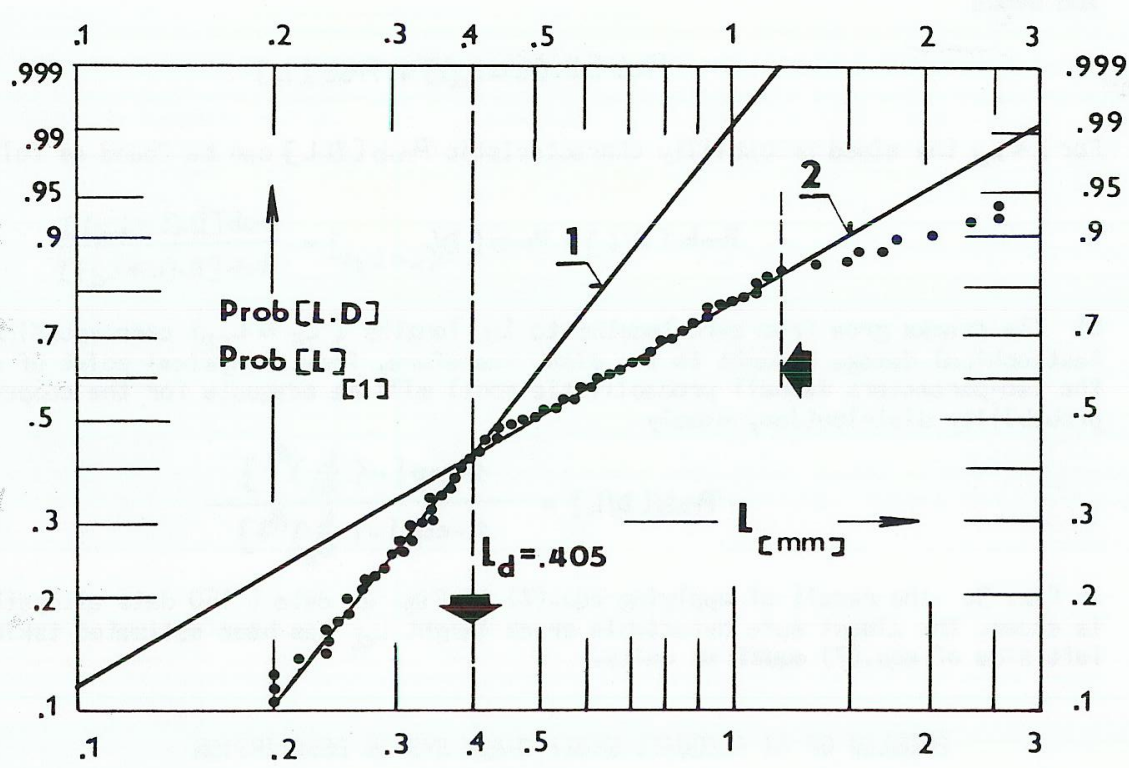

Fig. 2. Crack length first occurence experimental data $L$ as investigated in Weibull probability paper in respect to human operator s ability to crack detection. 1,2 component probability distribut lons $/$ see right side of equ.(6), sample upper limit due to an a prior agreed test unit number $\mathbb{N}$. 
where Prob [D.L] is the joint occurence probablility of the event that a crack is dotected at its first occurence and has a length of $L \mathrm{~mm}$ Prob $[D / L]$ means the ditional probability of a crack being detected given its length [L. Prob [L] means the probability of a crack having a lenght $y<L$ mm independently of $[L]$ tection method used. It is evident that the conditional probabllity Prob [D/I] presents an adequate characteristic to be found as qualifying the in in ity level of are man operator in respect to crack detection. From equ.(2), we obtain the basic formula

$$
\text { Prob }[D / L]=\frac{\text { Prob }[D \cdot L]}{\text { Prob }[L]}
$$

For est imat ing Prob $[D / L]$ from experimental crack length data, see $\mathrm{Flg}$. 2., two basic physical boundary conditions are to be taken into account : A) Given an almost sure detectable crack length $L_{d}$, then for all crack lengths

$$
\operatorname{Prob}\left[D /\left(L \geq L_{d}\right)\right]=1,0
$$

and hence

$$
\operatorname{Prob}\left[D \cdot\left(L \geq L_{d}\right)\right]=\operatorname{Prob}[L]
$$

For $L<L_{d}$ the aimed probability characteristic Prob $[D . L]$ can be iound as follows

$$
\operatorname{Prob}[D / L] \equiv \operatorname{Prob}\left[D /\left(L<L_{d}\right)\right]=\frac{\operatorname{Prob}\left[D \cdot\left(L<L_{d}\right)\right]}{\operatorname{Prob}\left[D \cdot\left(L \geq L_{d}\right)\right]}
$$

B) The cracks grow from zero iongths to $L_{F}$ lengths $\left(L_{F} \equiv L_{C R}\right)$ corresponding to catastrophical damage brought to the disk. Therefore, from a physical point of view, the two-parameters Weibull probabilistic model will be adequate for the component probability distribution, namely

$$
\operatorname{Prob}[D / L]=\frac{1-\exp \left\{-\left(\frac{L}{\theta_{1}}\right)^{B_{1}}\right\}}{1-\exp \left\{-\left(\frac{L}{\theta_{2}}\right)^{B_{2}}\right\}}
$$

In Fig. 3. the result of applying equ. (7) to Fig. 2. data ( 160 data altogether is shown. The almost sure detectable crack lenght $L_{d}$ has been est imated taking the

\section{PROBLEM OF AN ADEQUATE SHORT CRACK SYSTEM DESCRIPTION}

Drexler, Statezny' ( 1983 ) presented one of the possible assessments of this problen using the quantile crack length $L_{Q}$ for $Q=0.05$ and the instant aneous total number of cracks $n_{c}$ in the fir-tree bl ade at tachments of the disk as parameters describing the short crack system as a whole. In Fig. I. , the $n_{c}$ total number of cracks is reerred to the programme test unit number by the following formula

$$
n_{c}=(m+1) \cdot\left(1-\exp \left\{-\left(\frac{N-N_{0}}{\theta}\right)^{\alpha}\right\}\right)
$$

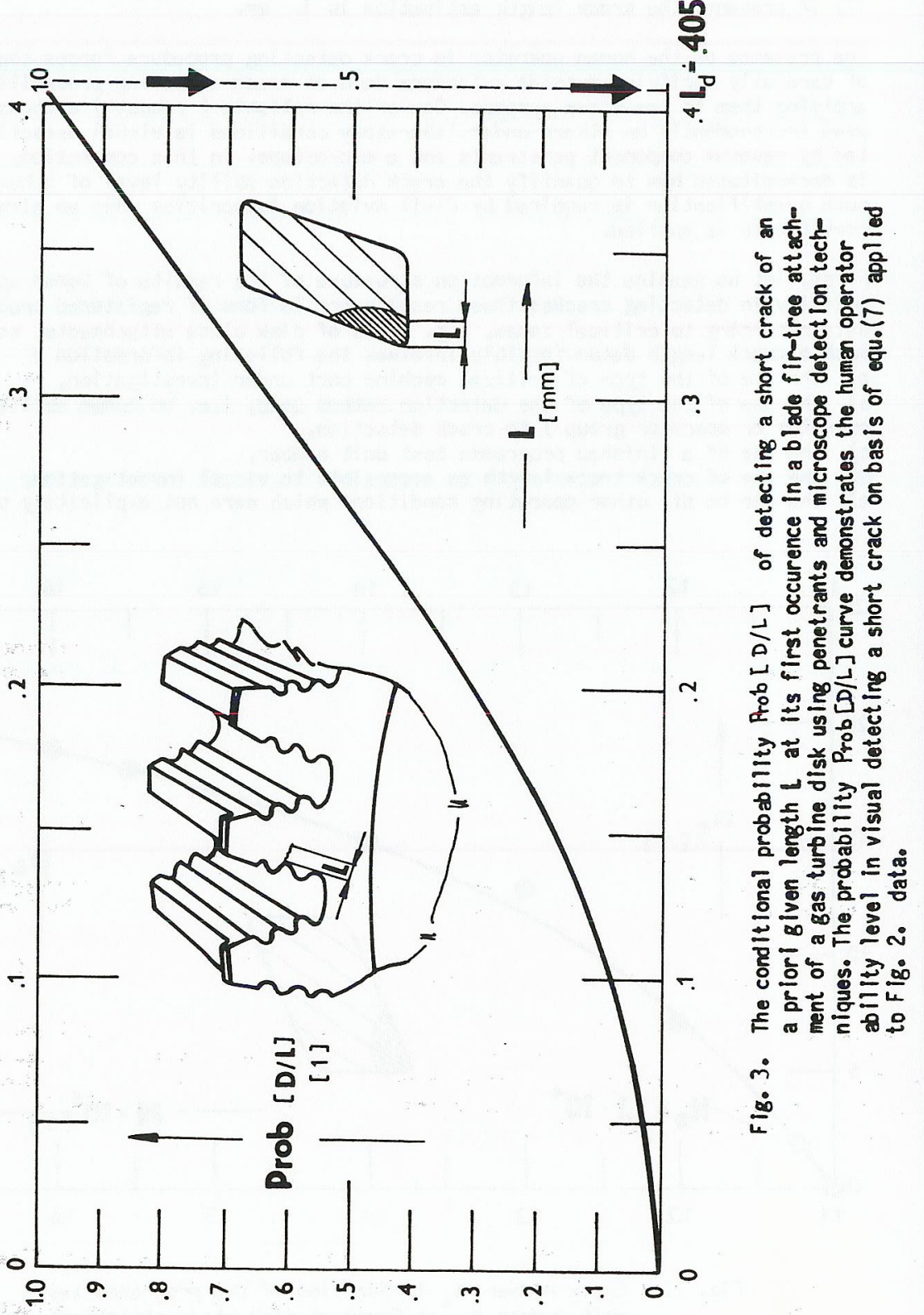


$m$ being the number of fir-tree attachments to the disk under investigation, whereby the erack occurence probability in one of the blade at tachments is given by

$$
P_{a}=\frac{n_{F}}{m+1}
$$

PROBLEM OF ESTIMATING LIFE TO SAFE CRACK OCCURENCE

Referring to airworthiness requirements for a hazard rate the life est imation in test unit number to safe crack occurence within a single disk has been derived by Němec, Drexler (1984) as follows

$$
N_{F S C} \geq \Lambda^{-1} \cdot\left\{-\ln \cdot\left(1-\operatorname{Prob}\left[n_{F S C} / n_{F} ; m ; M=1\right]\right)\right\}
$$

where $n_{F}, n_{F S C}$ are total numbers of cracks in the disk blade fir-tree attachments up to the safe crack length $L_{F S C}$ and to the critical length $L_{C R}, M$ is the number of disks accounted for in the life estimation, whereby the conditional probability

$$
\begin{aligned}
\operatorname{Prob}\left[n_{F S C} / n_{F} ; m ; M=1\right]-\frac{\operatorname{Prob}\left[\left(x<n_{F S C}\right) \cdot n_{F} ; m ; M=1\right]}{\operatorname{Prob}\left[x<n_{F} ; n_{F} ; m ; M=1\right]} & \\
& =\frac{\sum_{j=1}^{n_{F S C}} c_{j}^{m} \cdot p_{a}^{j} \cdot\left(1-p_{Q}\right)^{m-j}}{\sum_{j=1}^{n_{F}} c_{j}^{m} \cdot p_{a}^{j} \cdot\left(1-p_{a}\right)^{m-j}}
\end{aligned}
$$

Considering the $M \gg 1$ disk production series, the probability (11) of meeting $x<n_{\text {Ec }}$ cracked ones of $m$ fir-tre blade attachments in the least reliable one of $M$ produced disks changes to

$$
\begin{aligned}
& \operatorname{Prob}\left[n_{F S C} / n_{F} ; m ; M \gg 1\right]=1-\left(1-P_{r o b}\left[n_{F S C} / n_{F}\right.\right. \\
& m ; M=1])^{M}=1-\exp \left\{-M \cdot \operatorname{Prob}\left[\left(x<n_{F S c} n_{F} ; m ; M=1\right]\right\}\right.
\end{aligned}
$$

wher eby Prob $\left[x<n_{F} ; n_{F} ; m ; M \gg 1\right]$ approaches unity. Hence, the life est imation $N_{F S C}(M)$ to safe crack occurence for the least reliable one of $M$ disks is given by the formula

$$
N_{F S C}(M) \geq \Lambda^{-1} \cdot M \text {. Prob }\left[\left(x<n_{F S C}\right) \cdot n_{F} ; m ; M=1\right]
$$

In our example case, we had $\Lambda=1.10^{-8}, n_{F}=24, m=28,1 \leq M \leq 5000$ and for $P_{a}=0.82759$. The result when apply ing equ.(13) to these data is shown in Fig. 4. Therefrom we see that production series increase in $M$ from 1 to 5000 disks diminishes in our example case the possible programme test unit number gain $\Delta N=N_{F s c}-N_{0}$ to about a half, when $\mathrm{N}_{0}=1.1 \times 10^{4}$.

\section{REFERENCES}

Drexler, J., and J. Statečný (1983). A contribution to airworthiness certification of gas turbine disks. Proceedings ISABE ( paper 83-7071), Paris

Němec, J., and J. DrexTer (1984). Short crack development in mechanical structures. Proceedings ICF6, New Delhi.

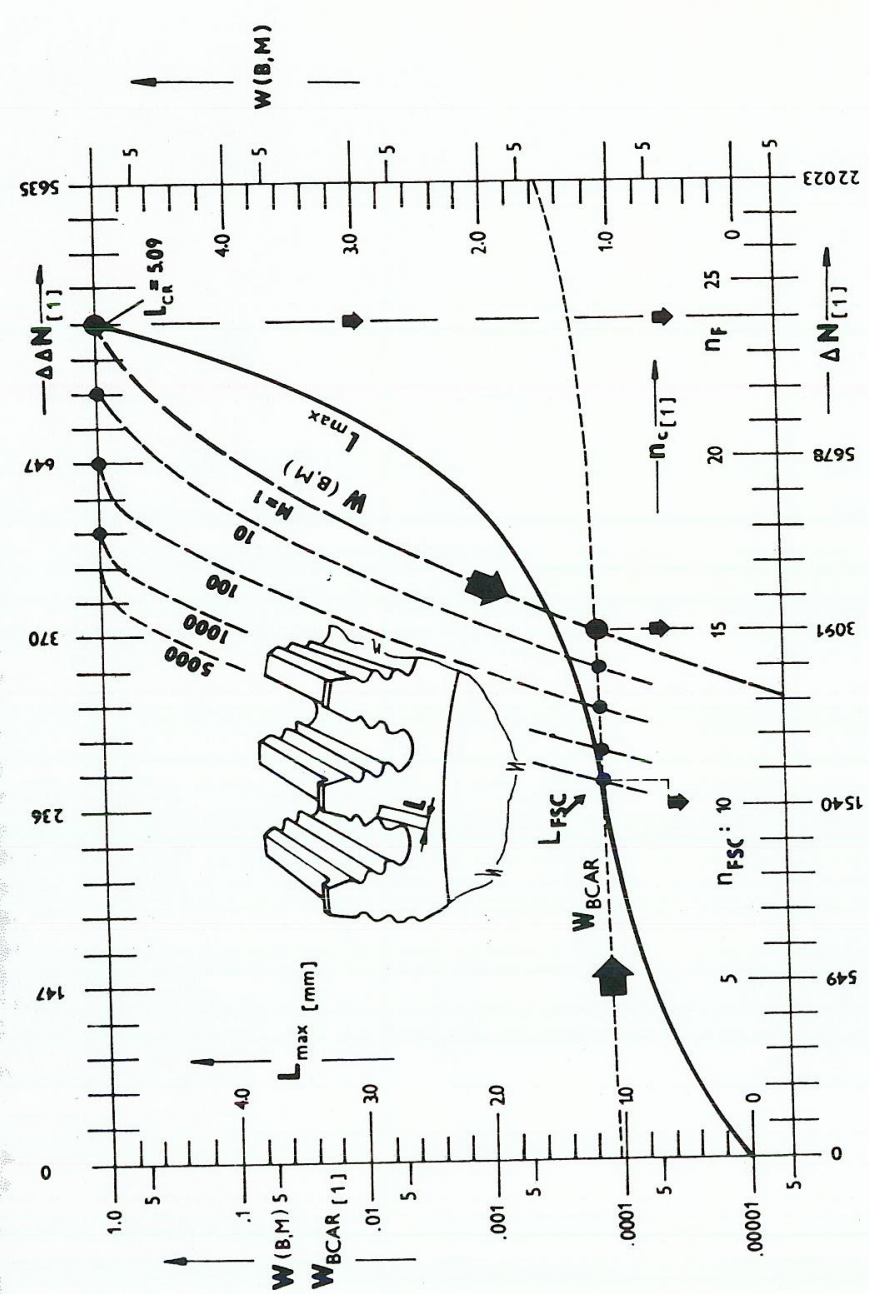

-

E

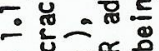
"Nu

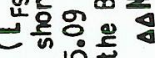

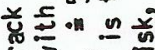
¿

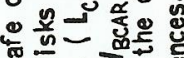
का $\infty \sum$ MN 我

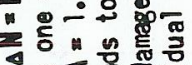
$\leq \div<\frac{0}{0}$

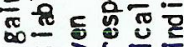
인은 若的落

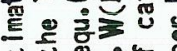

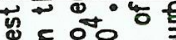
吅 象 论 $\stackrel{\therefore}{\dot{0}}$ 